InIFolPharm

Antikoagulation

\title{
Rivaroxaban - therapeutisches Potenzial noch nicht ausgeschöpft?
}

Die Antikoagulation blickt auf eine Erfolgsgeschichte zurück, die in den 1930erJahren ihren Anfang nahm und in der Entwicklung der modernen Antikoagulantien mündete. Inzwischen sind die modernen Wirkstoffe in verschiedenen Indikationen zugelassen, wobei der direkte Faktor-Xa-Hemmer Rivaroxaban $\left(\mathrm{Xarelto}^{\circledR}\right)$ das breiteste Indikationsspektrum besitzt. Es reicht von der Prophylaxe venöser Thromboembolien nach elektiver Knie- und Hüftgelenksersatzoperation über die Schlaganfallprophylaxe bei Vorhofflimmern und die Behandlung und Sekundärprophylaxe tiefer Venenthrombosen bis hin zur Behandlung und Sekundärprophylaxe von Lungenembolien.

In dieser Indikation gab es für Rivaroxaban jüngst eine Zulassungserweiterung, nachdem in der EINSTEIN-PE-Studie bei mehr als 4.800 Patienten gezeigt wurde, dass die Substanz ebenso wirksam ist wie die bisherige Standardtherapie, allerdings signifikant weniger schwere Blutungen bedingt. So betrug die Inzidenzrate schwerer Blutungen lediglich 1,1\% gegenüber 2,2\% unter der Standardtherapie, berichtete Dr. Frank Misselwitz, Wuppertal. Ein weiterer Vorteil besteht darin, dass mit nur einem einzigen Wirkstoff Therapie wie auch Sekundärprophylaxe zu realisieren sind, sagte der Mediziner, der maßgeblich an der Entwicklung von Rivaroxaban beteiligt war. $\mathrm{Zu}$ erwarten ist aus seiner Sicht eine weitere Zulassung für den Faktor-Xa-Hemmer, da dieser auch bei der Sekundärprävention des akuten Koronarsyndroms bereits seine klinische Wirksamkeit und Sicherheit in Studien unter Beweis gestellt hat.

Christine Vetter, freie Medizinjournalistin

Pressegespräch „Antithrombose-Forschung auf der Höhe der Zeit", anlässlich der 57. Jahrestagung der Gesellschaft für Thrombose- und Hämostaseforschung (GTH), München, 22.2.2013 Veranstalter: Bayer Healthcare

\section{Volkskrankheiten durch personalisierte Ernährung aufhalten}

Ehrgeiziges Ziel des neuen Nestlé Institute of Health Sciences (NIHS) in Lausanne ist die Entwicklung wissenschaftlich fundierter Ernährungskonzepte auf personalisierter Basis, um die Entwicklung von Volkskrankheiten wie Diabetes mellitus oder Alzheimer-Demenz zu verzögern, informierte Dr. Emmanuel E. Baetge, wissenschaftlicher Leiter des NIHS.

Im NIHS werden bis zu 150 Wissenschaftler unterschiedlicher Fachrichtungen arbeiten. Die Forschungsarbeiten konzentrieren sich schwerpunktmäßig auf „metabolische Gesundheit“, „gastrointestinale Gesundheit" und „Hirn-Gesundheit". Besondere Herausforderung ist die Demenz: Die Diagnose wird erst in einem späten Krankheitsstadium gestellt, wenn das kognitive Defizit aufgrund irreparabler zerebraler Läsionen schon relativ weit fortgeschritten ist. Dabei setzen die letztlich zur zerebralen Degeneration führenden biologischen Prozesse 10- 20 Jahre vor Manifestation der ersten klinischen Symptome ein. Bereits zu diesem frühen Zeitpunkt müssten präventive Strategien initiiert werden, um den kognitiven Verlust aufzuhalten. Derzeit laufen am NIHS Longitudinaluntersuchungen an gesunden Personen, um massenspektrometrisch potenzielle molekulare Marker für eine präklinische Demenz zu identifizieren. Des Weiteren werden in humanen Zellkulturen Naturprodukte und Nahrungsbestandteile auf ihre Fähigkeit getestet, um Demenzerkrankungen verzögern oder besser behandeln zu können.

Dr. Katharina Arnheim, freie Medizinjournalistin

Pressekonferenz und Eröffnung des Nestlé Institute of Health Sciences, Lausanne, 2.11.2012, Veranstalter: Nestlé
Neuropathische Schmerzen

Beim neuropathischen Rückenschmerz kann Pregabalin (Lyrica ${ }^{\circledR}$ im Rahmen eines differenzierten Therapieansatzes ein Mittel der Wahl sein. Die Vielfalt der Phänotypen erschwert jedoch oftmals die Diagnose. Mit painDETECT ${ }^{\circledast}$ steht ein einfaches Screening-Instrument zur Verfügung, mit dessen Hilfe sich neuropathische Schmerzkomponenten gut erfassen lassen: Der validierte Fragebogen, der vom Patienten in ungefähr fünf Minuten ausgefüllt werden kann, erfasst Schmerzintensität, -muster und -qualität. Neuropathische Schmerzkomponenten werden mit einer Sensitivität und Spezifität von über $80 \%$ detektiert.

Nach Informationen von Pfizer

\section{Felix-Jerusalem-Preis}

Im Rahmen ihres 21. Jahreskongresses hat der wissenschaftliche Beirat der Deutschen Gesellschaft für Muskelkranke e. V. (DGM) den Felix-Jerusalem-Preis zur Förderung der Forschung auf dem Gebiet der neuromuskulären Erkrankungen an Dr. Frédéric Chevessier, Neuropathologisches Institut, Universitätsklinikum Erlangen, verliehen. Der mit 7.500€ dotierte Forschungspreis wurde in diesem Jahr von der Firma Genzyme gesponsert.

Nach Informationen von Sanofi-Genzyme

\section{Schizophrenie und bipolare Störungen}

Für die Behandlung schizophrener Psychosen und bipolarer Störungen steht mit Ziprasidon-Actavis Hartkapseln ab sofort eine bioäquivalente Alternative zum Erstanbieterprodukt zur Verfügung, die eine erhebliche Budgetentlastung ermöglicht. Ziprasidon-Actavis Hartkapseln sind in den Wirkstärken $20 \mathrm{mg}, 40 \mathrm{mg}, 60 \mathrm{mg}$ und $80 \mathrm{mg}$ in der Blisterpackung mit 30 oder 100 Hartkapseln erhältlich. Zudem bietet Actavis eine Schizophrenie-Broschüre für Angehörige an, die bestellt werden kann unter: info.de@actavis.de.

Nach Informationen von Actavis 